Received: September 05, 2016

Accepted: December 12, 2016

Published: June 13, 2017

\section{Polyvinyl Alcohol-Nanobioceramic Based Drug Delivery System}

\author{
Shameem Shaik ${ }^{1}$, Seethalakshmi ${ }^{2 *}$, Kaviya $\mathbf{M}^{1}$, Venkatachalapathy ${ }^{3}$, Sridhar TM $^{1}$ \\ ${ }^{1}$ Department of Analytical Chemistry, University of Madras, Guindy Campus, Chennai, \\ India \\ ${ }^{2}$ Department of Chemistry, Rajalakshmi Engineering College, Chennai, India \\ ${ }^{3}$ Department of Chemistry, SRM Eswari Engineering College, Chennai, India
}

*Corresponding author: Seethalakshmi K, Research Scholar, Department of Chemistry, Rajalakshmi Engineering College, Thandalam, Chennai, India, E-mail: js200108@gmail.com

\section{Abstract}

Drug delivery research today is an advanced and important area in pharmaceutical research and application of nanotechnology includes enhancement of the solubility and permeability of the drugs so as to improve their bioavailability including delivery to the targeted site. Hydroxyapatite (HAP) based bioceramic nanoparticles composed of biodegradable polymers have been used in the present work to develop an amoxicillin based delivery systems. The synthesized n-HAP powders were estimated for the $\mathrm{Ca} / \mathrm{p}$ ratio. This ratio indicates the presence of HAP as a single phase. The nano structure, morphology and presence of vibrational groups are confirmed using instrumental analysis. The SEM images show the spherical shaped particles of nano hydroxyapatite are confirmed. The loading and unloading characteristics of the drug were recorded spectro photometrically.

\section{Keywords}

Nanofibres; Electrospinning; Polyvinyl Alcohol; Hydroxyapatite; Amoxicillin;

\section{Introduction}

Various technologies have been explored for different routes of administration such as oral, parenteral, nasal, dermal, vaginal, etc. Drug delivery is the method or process of administering a pharmaceutical compound to achieve a therapeutic effect in humans or animals. It has been reported that the ultimate thera- peutic and toxic effects produced by a drug, when administered as a solid dosage form and the resultant benefit or risk to a patient, are dependent on the sum of several interacting variables associated with the pharmacological properties of the drug, physiological and pathological factors associated with the patient and the physicochemical properties of the dosage form $[1,2]$. Several researchers are, therefore, engaged in exploring the physicochemical aspects of dosage forms and their relationship with therapeutic efficacy [3, 4]. Different types of drug delivery systems have been developed by manipulating the physicochemical properties such as particle size, compound lipophilicity, surface charge, etc. Several products are available in the market based on these technologies and a large number of products are in clinical trials but they are yet to reach the patients. In this context, nanotechnology has been shown to provide superior drug delivery systems for a better management and treatment of cancer and other diseases like AIDS, tuberculosis and malaria. Current efforts in the area of drug delivery include the development of targeted delivery in which the drug is only active in the target area of the body and sustained release formulations in which the drug is released over a period of time in a controlled manner from a formulation. The application of nanotechnology in drug delivery research includes enhancement of the solubility and permeability of the drugs so as to improve their bioavailability including delivery to the targeted site like for example malignant tumour. For the treatment of human diseases, nasal and pulmonary routes of drug delivery are gaining increasing importance. These routes provide promising alternatives to parenteral drug delivery particularly for peptide and protein therapeutics. For this purpose, several drug delivery systems have been formulated and are being investigated for nasal and pulmonary delivery. These include liposomes, proliposomes, microspheres, gels, prodrugs, cyclodextrins, among others. biocompatibility, targeting of specific sites 
or cell populations in the lung, release of the drug in a predetermined manner, and degradation within an acceptable period of time. In order to achieve efficient targeted delivery, the designed system must avoid the host's defence mechanisms and circulate to its intended site of action. Types of sustained release formulations include liposomes based drug loaded biodegradable microspheres and drug polymer conjugates.

Nanoparticles (NPs) are defined as particulate dispersions or solid solutions with a size range of 10-300 nm. Nanoparticle drugs are designed in such a way that the drug is dissolved, entrapped, encapsulated or attached to a nanoparticle matrix [5]. The preparation of nanoparticles depends on the process and the end product, namely nanospheres, nanocapsules and nanocomposites. Nanocapsules (NCs) are systems in which the drug is loaded inside the cavity enclosed by a unique polymer membrane. Nanospheres are complex matrix systems in which the drug is adsorbed, dissolved or dispersed throughout the matrix system [6, 7]. Nanocomposites are a multiphase material (usually a mixture of two or more polymers) where one of the phases has one, two or three dimensions of nanometer size.

Bioceramics has evolved as an integral and vital segment of our modern health-care delivery system. The full potential is yet to be explored in the years to come. Hydroxyapatite Ca10(PO4)6(OH)2 is a well-established biocompatibile ceramic capable of forming a strong chemical bond with natural bone. It is chemically $(\mathrm{Ca} / \mathrm{P}$ ratio 1.66$)$ and mineralogically identical with normal human hard tissue- bone and would be stable in contact with body fluids. In the present work nano hydroxyapatite (n-HAP) was synthesized and mixed with electrospun PVA and loaded with amoxillin drug and profile its release.

\section{Materials and Methods}

\subsection{Materials}

The following reagents and chemicals used in the experiment were of analytical grade. Calcium nitrate, Cetyl Tri methyl Ammonium Bromide (CTAB), Ammonium hydrogen phosphate, Ammonia (for maintaining $\mathrm{pH}$ ), Ethanol, Amoxicillin trihydrate drug, Sodium chloride Phosphate buffer. Polyvinyl alcohol, Average MW equal to 85, 000-124,000 form Sigma Aldrich were used for electrospining.

\subsection{Method}

\subsubsection{Synthesis}

\subsubsection{Synthesis of nanohydroxyapatite(n-HAP)}

Nanohydroxyapatite was prepared by precipitation method. $1 \mathrm{M}$ calcium nitrate was prepared and taken in a $500 \mathrm{ml}$ beaker and maintained the $\mathrm{pH}-11$ by adding ammonia and stirred it for 30 minutes. $0.01 \mathrm{~mol}$ CTAB was added to the beaker and stirred it for $1 \mathrm{~h}$ then $0.67 \mathrm{M}$ of ammonium hydrogen phosphate was added slowly and maintained $\mathrm{pH}-10$ by adding ammonia with stirring for $2 \mathrm{hrs}$, until the precipitate was formed. Aging the precipitate for $6 \mathrm{~h}$ after that wash the filtered precipitate with ethanol and kept in oven at $80^{\circ} \mathrm{C}$ for $1 \mathrm{~h}$. The dried precipitates are crushed into powder form and kept it in the muffle furnace at $800^{\circ} \mathrm{C}$ for 1 h. The amount of calcium present in the sample was estimated by complexometric titration using EDTA solution. The color change from wine red to blue indicates the end point using EBT indicator. From these values the amount of calcium present was obtained which was further used to calculate the $\mathrm{Ca} / \mathrm{P}$ molar ratios.

\subsubsection{Electrospinning of PVA}

Polymer ceramic nanocomposites were prepared using an electrospinning apparatus, indigenously designed and developed apparatus. The PVA incorporated with HAP was prepared by depositing HAP over nanofibrous membranes. The polymer solutions were extruded at a speed of $1 \mathrm{~mL} / \mathrm{h}$ by using a syringe pump (NE-300, New Era Pump Systems, Inc.) with the voltages at 20 KV. Aluminum foil was used as the collector.

\subsubsection{Drug Loading}

For drug loading (Amoxicillin trihydrate), $100 \mathrm{mg}$ of n-HAP and drug is loaded in a test tube and then add $4 \mathrm{ml}$ of $0.15 \mathrm{M}$ $\mathrm{NaCl}$ and stir it for $1 \mathrm{hr}$ at room temperature. The test tube was centrifuged at $2000 \mathrm{rpm} / \mathrm{min}$ for about $10 \mathrm{~min}$. $3 \mathrm{ml}$ supernatant was withdrawn for validation by UV-visible spectrophotometer at 200-400nm. Drug loading can be calculated by

Drug loading $(\% \mathrm{w} / \mathrm{w})=$ Drug incorporated/Dry powder weight after loading $\times 100$

Amoxicillin loaded n-HAP powder were compressed with electrospun PVA by a pressure of $25 \mathrm{~kg} / \mathrm{cm} 2$ (2tons) using a hydraulic press and mini pellets of size, $2 \mathrm{~mm} \times 2 \mathrm{~mm} \times 1 \mathrm{~mm}$ were prepared. Samples containing 5 mini pellets of each formulation were placed in a beaker containing $5 \mathrm{ml}$ of pH7.4 phosphate buffer saline at room temperature $[8,9]$. Elution fluids were replaced by fresh buffer at regular intervals. Removed elution fluids were collected for determination of drug concentration by UVvisible spectrophotometer at maximum wavelength at 200-400 $\mathrm{nm}$.

\subsection{Characterization}

The amount of phosphorous content present in the sample as a phosphomolybdo complex developed with molybdenum blue method using UV- Spectrophotometry. From the amount of calcium and phosphorous content present in the sample, the $\mathrm{Ca} / \mathrm{P}$ ratios were calculated and found to be 1.67 corresponding to that of n-HAP. Powder X-ray diffraction (XRD) patterns were obtained using an X-ray diffractometer equipped (Bruker model D8) using $\mathrm{Cu} \alpha$ radiation (wavelength $=\lambda-1.5406 \AA$ ) to identify the crystalline phase, peak positions were compared with standard files. SEM (Model S-4700, Hitachi) was used to study the morphologies of the powders. Diffuse Reflectance Spectroscopy (DRS) was performed on a Shimadzu UV-2450 spectrophotometer. FT-IR was recorded in ATR mode using UATR Perkin Elmer spectrum II.

\section{Results and Discussion}

The synthesized n-HAP powders were estimated for the $\mathrm{Ca} / \mathrm{P}$ ratio. The $\mathrm{Ca} / \mathrm{P}$ ratio of 1.66 corresponding to that of hydroxyapatite with the formula $\mathrm{Ca}_{10}\left(\mathrm{PO}_{4}\right)_{6}(\mathrm{OH})_{2}$ was obtained. This 
ratio indicates the presence of HAP as a single phase. The nano structure, morphology and presence of vibrational groups would be confirmed using instrumental analysis.

\subsection{X-Ray Diffraction (XRD)}

The XRD patterns of n-HAP powder after sintering at $800^{\circ} \mathrm{C}$ are shown in the figure 1. n-HAP powder has the corresponding hkl values (002), (211), (112), (300), (202), (310), (222), (213) and (004).

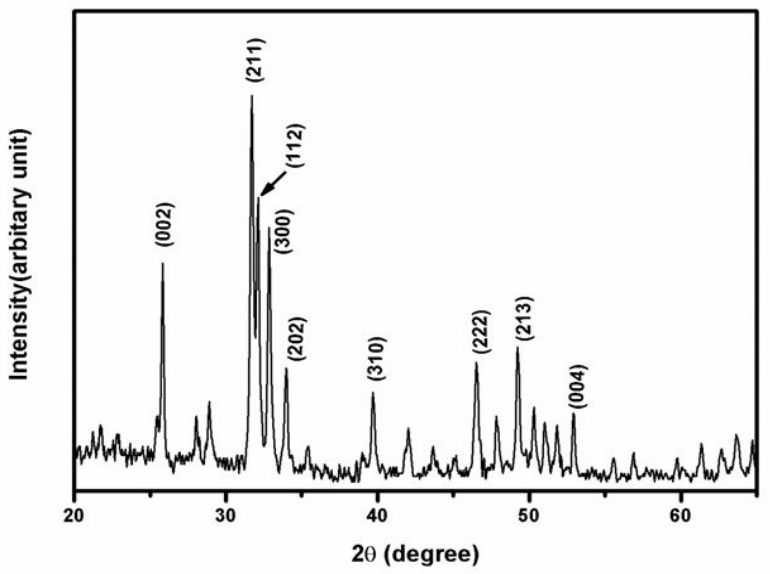

Figure 1: XRD Patterns of nano Hydroxyapatite powder

These hkl values of HAP indexing with JCPDS file no.09-0432 showed a minimal line broadening and sharp intense peak, indicating a well crystallite material [10-12]. The absence other calcium phosphate (Tricalcium phosphate) peaks were observed in the patterns. This confirms the presence of monophasic Hydroxyapatite in the material.

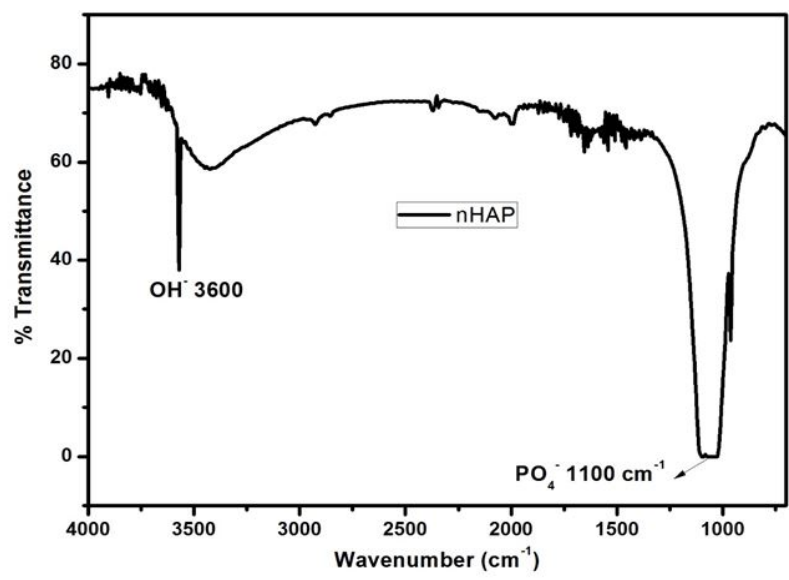

Figure 2: FTIR spectra of nano Hydroxyapatite powder

\subsection{FT Infrared Spectroscopy}

FT-IR patterns presented in above figure.2, confirm the formation of n-HAP at different temperatures of $100-800^{\circ} \mathrm{C}$. The spectra possessed an $(\mathrm{OH})^{-1}$ group in the region of $3600 \mathrm{~cm}-1$ and $\left(\mathrm{PO}^{4-}\right)$ group comes out from the region of $1100 \mathrm{~cm}^{-1}$. The presence of phosphate peak is due to $\mathrm{P}=\mathrm{O}$ stretching and hydroxide peak is due to stretching and bending vibrations. From this analysis the formation of n-HAP is confirmed [10].

The successful loading of amoxicillin onto the n-HAP surface was qualitatively confirmed by FTIR spectroscopy. In the figure. 3 the peak at $3570 \mathrm{~cm}-1$ is due to the stretching vibration of amino and hydroxyl group in amoxicillin structure. The absorption peak

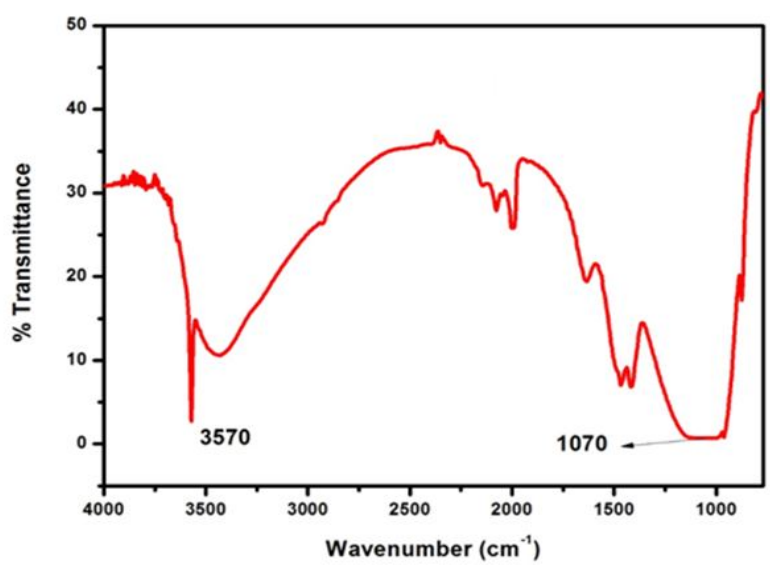

Figure 3: FTIR spectra of nano HAP- amoxicillin powder

at $1070 \mathrm{~cm}-1$ may be due to the stretching vibration of phosphate group. The FTIR data qualitatively verified the loading of amoxicillin onto the n-HAP surfaces.

\subsection{Morphological Analysis}

The SEM images of the nano hydroxyapatite indicate the presence of nano sized grain. The hydroxyapatite nano particles formed were highly agglomerated and are given in figure 4 . The

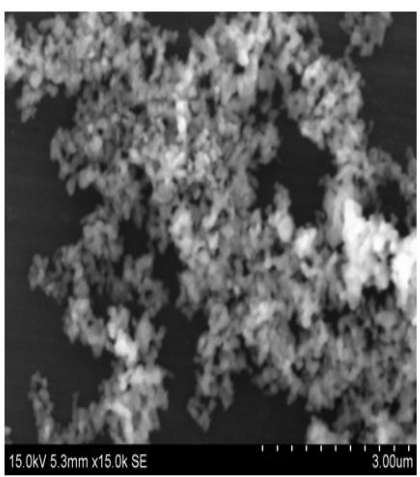

a

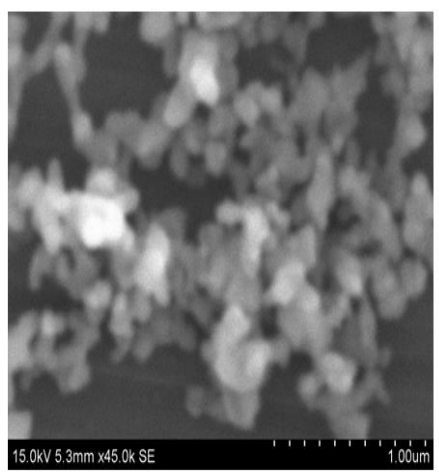

Figure 4: SEM micrographs of Nano hydroxyapatite The SEM micrographs of Nano hydroxyapatite is presented in Fig.4 a-b:

surface of the hydroxyapatite consists of tightly packed grains forming good adherence. The shaped particles with clumped distributions are visible from the SEM analysis. The size of the particles ranges from 50 to $160 \mathrm{~nm}$. The SEM images show the nano composite after drug loading in the PVA electrospun polymer with nHAP as given in figure 5 . The results reveal that the particles are 
a

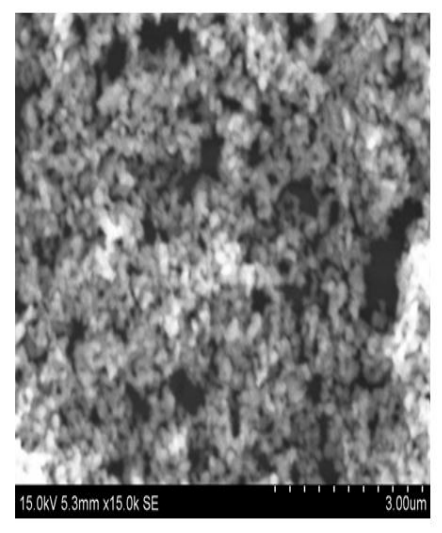

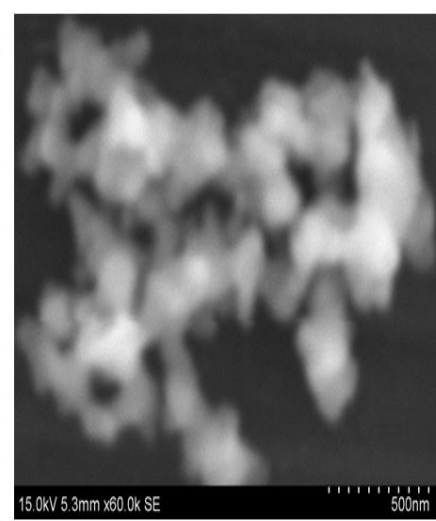

b
Figure 5: SEM micrographs of Nano hydroxyapatite loaded with amoxicillin drug

Table 1: Percentage of Drug Loading into the N-HAP and PVA Matrix from UV-Vis Absorption Spectra Drug loading $(\% \mathrm{w} / \mathrm{w})=$ Drug incorporated/Dry powder weight after loading $\times 100$

\begin{tabular}{|c|c|}
\hline \multicolumn{2}{|c|}{ 100 mg of amoxicillin with n-HAP, PVA } \\
\hline Time (hr) & Calculation (\%w/w) \\
\hline 1 & 66.66 \\
\hline 2 & 30.67 \\
\hline 24 & 44.05 \\
\hline 48 & 31.34 \\
\hline
\end{tabular}

of random spherical shape with a moderate rough surface indicating that the drug is homogeneously dispersed with the polymeric matrix. The SEM image reveals that the nano composites formed are of narrow size distribution of 200 to $240 \mathrm{~nm}$. The data further confirms that nano hydroxyapatite has been loaded with amoxicillin drug.

\subsection{Spectrophotmetic estimation of drug release}

The UV spectrum of the PVA, HAP and drug systems is given in figure. 6-9.

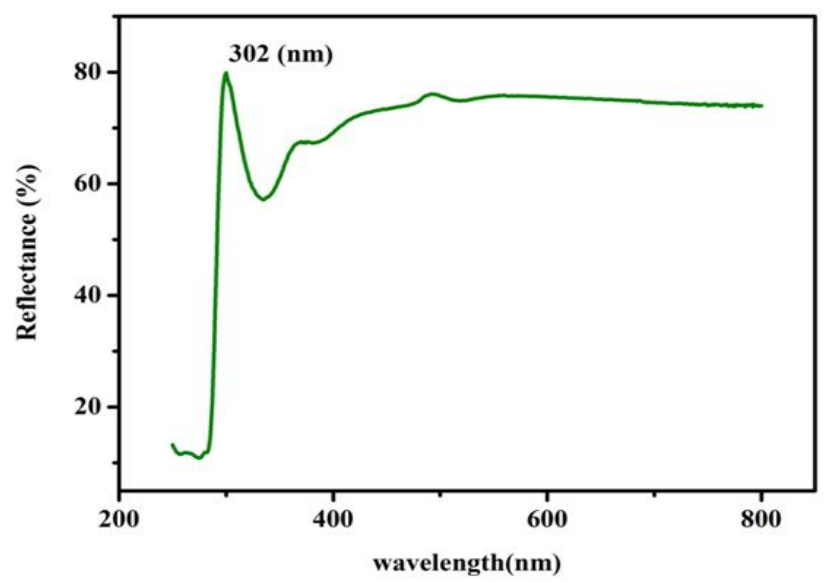

Figure 6: UV visible spectra of amoxicillin drug containing PVA

figure. 6 and 7 shows the reflection edge wavelengths of PVA

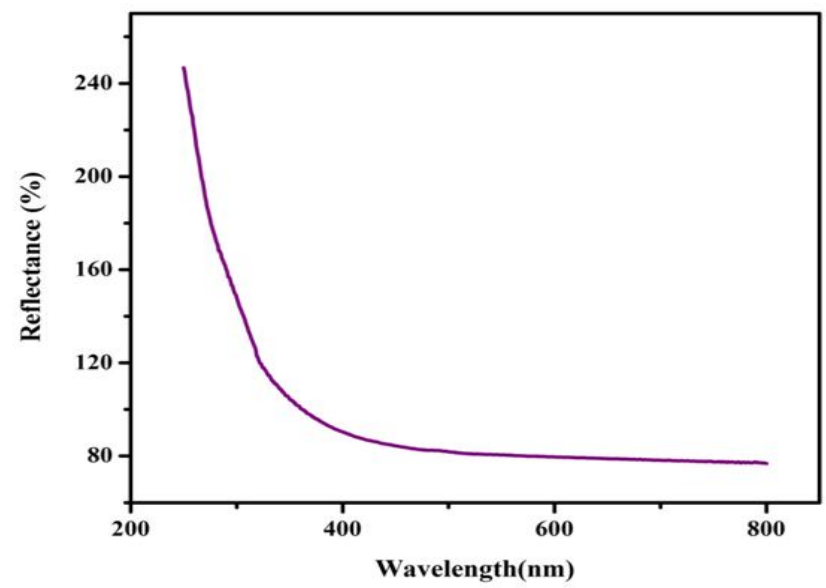

Figure 7: UV visible spectra of amoxicillin drug containing n-HAP and PVA

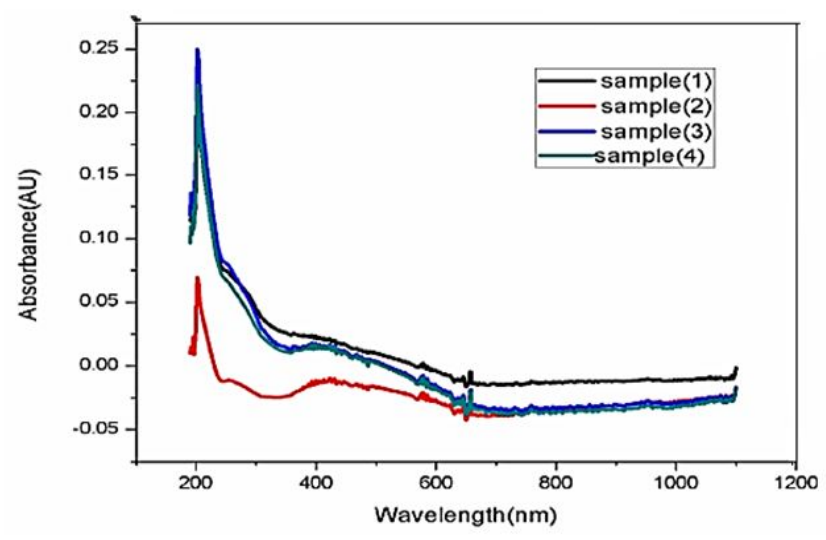

Figure 8: UV visible spectra of amoxicillin drug release in n-HAP with PVA for $22 \mathrm{~h}$

and amoxicillin were in the range of 340-400 $\mathrm{nm}$ where as the of n-HAP was smaller than $250 \mathrm{~nm}$. In case of both n-HAP and amoxicillin and n-HAP, PVA and drug the reflection in the UVVis range was imperfect and a small absorption in this range is observed. Moreover, it may have had a correlation between the shift of binding energy and the shift of band gap energy of the materials. The above results confirmed the loading of the drug into the n-HAP-PVA matrix.

The drug loading profiles for n-HAP-PVA with amoxicillin with respect to loading time have been calculated and the results given in Table 1. figure. 6-7 shows that the amount of drug is loaded at 200-400 nm [12, 13]. But, better loading is observed at 277 $\mathrm{nm}$ as shown which indicates it is a time independent process. This confirms that the amount of drug that has been loaded in the above regions.

\subsection{Drug release in n-HAP and PVA by mini pellet technique}

The release kinetics of amoxicillin/n-HAP/PVA was determined by recording the absorbance of amoxicillin and PVA at $204 \mathrm{~nm}$ using a UV-visible spectrophotometer. The accumulated release of amoxicillin was calculated based on a standard amoxicillin absorbance concentration curve at $204 \mathrm{~nm}$. 


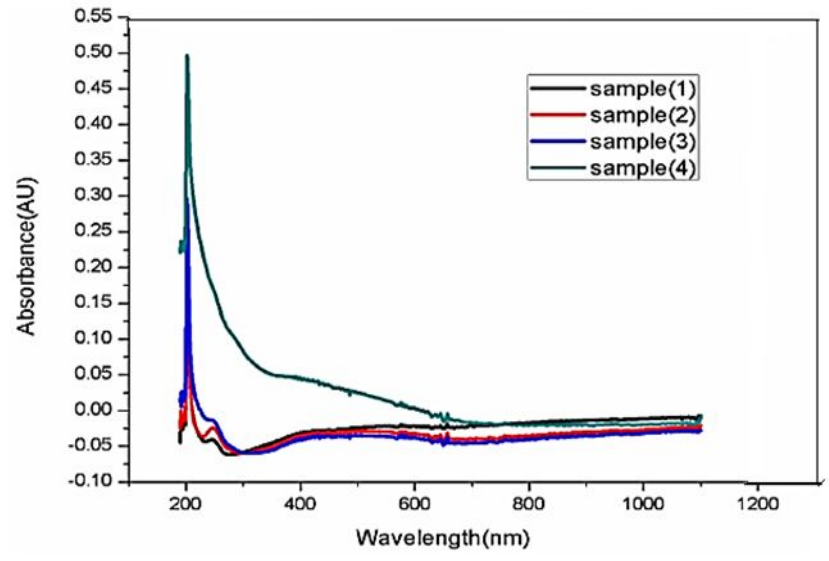

Figure 9: UV visible spectra of amoxicillin drug release in n-HAP with PVA for $48 \mathrm{~h}$

Table 2: Amoxicillin drug release in n-HAP at $22 \mathrm{~h}$ and $48 \mathrm{~h}$ obtained by UV-visible spectra

\begin{tabular}{|c|c|c|c|c|c|}
\hline \multirow{2}{*}{ Time } & $\begin{array}{c}\text { Elution } \\
\text { of } \\
\text { drug }\end{array}$ & & \multirow{2}{*}{ Time } & $\begin{array}{c}\text { Elution } \\
\text { of } \\
\text { drug }\end{array}$ & \\
\cline { 2 - 3 } & $\begin{array}{c}\text { Absor- } \\
\text { bance } \\
\text { (au) }\end{array}$ & $\begin{array}{c}\text { Wave- } \\
\text { length } \\
\text { (nm) }\end{array}$ & $\mathbf{4 8 ~ h}$ & $\begin{array}{c}\text { Absor- } \\
\text { bance } \\
\text { (au) }\end{array}$ & $\begin{array}{c}\text { Wave- } \\
\text { length } \\
\text { (nm) }\end{array}$ \\
\hline Sample 1 & 0.17 & 202 & Sample 1 & 0.09 & 202 \\
\hline Sample 2 & 0.07 & 202 & Sample 2 & 0.09 & 202 \\
\hline Sample 3 & 0.25 & 202 & Sample 3 & 0.29 & 202 \\
\hline Sample 4 & 0.22 & 202 & Sample 4 & 0.5 & 202 \\
\hline
\end{tabular}

The release kinetics of amoxicillin/n-HAP was determined by recording the absorbance of amoxicillin at $202 \mathrm{~nm}$ using a UVvisible spectrophotometer. The accumulated release of amoxicillin was calculated based on a standard amoxicillin absorbance concentration curve at $202 \mathrm{~nm}$. The elution studies indicate that the drug elution occurs at a maximum of $204 \mathrm{~nm}$. Adsorption of drug amoxicillin on n-HAP and PVA is time independent process. Drug release has been successfully carried out using minipellet technique. The release kinetics were carried out between 200$400 \mathrm{~nm}$. Further studies are needed to understand the percentage of doses of drug uptake and release with time and changes in composition n-HAP-PVA matrix.

\section{Conclusion}

In conclusion, we have designed and synthesized of nano hydroxyapatite by precipitation method in pure form. A Ca/P ratio of 1.67 was confirmed using the crystalline patterns recorded by $\mathrm{XRD}$ and vibrational bands with IR spectrum. SEM analysis indicates the change in the morphology between n-HAP and n-HAP. The drug adsorption and release kinetics of n-HAP and amoxicillin and PVA electrospun fibers were studied by UV analyses. Validation by UV-visible spectrophotometer was done at a $\lambda_{\max }$ of $208 \mathrm{~nm}$. The elution studies indicate that the drug elution occurs at a maximum of $204 \mathrm{~nm}$. Adsorption of drug amoxicillin on n-
HAP and PVA is time independent process. The results obtained indicate that drug loading by n-HAP and PVA is a time dependent process. The drug release mainly depends on its relation from the substrate with respective to time. The drug loading techniques reported are important for tissue engineering and pharmaceutical science.

\section{Acknowledgement}

The corresponding author express thanks to UGC (Ref.noMRP-6089(SERO/UGC)) for the financial assistance supported to this research work.

\section{References}

1. Lambert HP, and O'Grady FW. Antibiotic and Chemotherapy, 6th Edn., Churchill Living stone, Edinburgh. 1992;245-262.

2. Mylotte JM, Ksiazek S, Bentley. Rational approach to the antibiotic treatment of pneumonia in elderly. Drug \& Aging. 1994;4(1):21-33.

3. Shen $X, X u$ Q, Xu S, Li J, Zhang N, Zhang L. Preparation and transdermal diffusion evaluation of the prazosin hydrochloride-loaded electrospun poly (vinyl alcohol) fiber mats. J Nanosci Nanotechnol. 2014;14(7):5258-5265.

4. Alphandary HP, Andremont A \& Couvreur P. Targeted delivery of antibiotics using liposomes and nanoparticles: research and applications. International Journal of Antimicrobial Agents. 2000;13(3):155-168.

5. Xu F, Weng B, Gilkerson R, Materon LA, Lozano K. Development of tannic acid/chitosan/pullulan composite nanofibers from aqueous solution for potential applications as wound dressing. Carbohydr Polym. 2015;115:16-24.

6. Wang J, An Q, Li D, Wu T, Chen W, Sun B, et al. Heparin and vascular endothelial growth factor loaded poly (L-lactideco-caprolactone) nanofiber covered stent-graft for aneurysm treatment. J Biomed Nanotechnol. 2015;11(11):1947-1960.

7. Samit K Nandi, Biswanath Kundu, Samir K Ghosh, Tapan K Mandal, Someswar Datta, Dipak K De, et al. Cefuroximeimpregnated calcium phosphates as an implantable delivery system in experimental osteomyelitis. Ceramics International. 2009;35(4):1367-1376.

8. Nayak Amit Kumar and Sen Kalyan Kumar. Hydroxyapatite ciprofloxacin minipellets for bone-implant delivery: Preparation, characterization, in-vitro drug adsorption and dissolution studies Int J Drug Dev \& Res. 2009;1(1):47-59.

9. Amit Kumar Nayak, Bibek laha, kalyan kumar Sen. "Development of hydroxyapatite-ciprofloxacin bone-implants using Quality by design". Acta Pharm. 2011;61(1):25-36. doi: 10.2478/v10007-011-0002-x 
10. Kamachi Mudali U, Sridhar TM, Baldev Raj. 'Corrosion of Bio Implants' Sādhanā, (Academy Proceedings in Engineering Sciences, Indian Academy of Sciences). 2003;28(3-4):601-637.

11. Zhiping Zhang, Songwei Tan, Si-Shen Feng. "Vitamin E TPGS as a molecular biomaterial for drug delivery", Biomaterials. 2012;33(19):4889-4906. doi: 10.1016/j.biomaterials.2012.03.046

12. Matthew J Webber, John B Matson, Vibha K Tamboli, Samuel I Stupp. Controlled release of dexamethasone from peptide nanofiber gels to modulate inflammatory response. Biomaterials. 2012;33(28):6823-6832. doi: 10.1016/j.biomaterials.2012.06.003

13. uyin Zheng, Shige Wang, Shihui Wen, Mingwu Shen, Meifang Zhu, Xiangyang Shi. Characterization and antibacterial activity of amoxicillin-loaded electrospun nano-hydroxyapatite/poly(lactic-co-glycolic acid) composite nanofibers. Biomaterials. 2013;34(4):1402-1412. doi: 10.1016/j.biomaterials.2012.10.071

14. Amit Kumar Nayak, Ajoy Bhattacharyya, and Kalyan Kumar Sen. In Vivo Ciprofloxacin Release from HydroxyapatiteBased Bone Implants in Rabbit Tibia. 2011.

15. Ji W, Yang F, Ma J, Bouma MJ, Boerman OC, Chen Z, et al. Incorporation of stromal cell-derived factor- $1 \alpha$ in PCL/gelatin electrospun membranes for guided bone regeneration. Biomaterials. 2013;34(3):735-745. doi: 10.1016/j.biomaterials.2012.10.016

16. Su Y, Su Q, Liu W, Lim M, Venugopal JR, Mo X, et al. Controlled release of bone morphogenetic protein 2 and dexamethasone loaded in core-shell PLLACL-collagen fibers for use in bone tissue engineering. Acta Biomater. 2012;8(2):763-771. doi: 10.1016/j.actbio.2011.11.002
17. Zhiping Zhang Songwei Tan, Si-Shen Feng. Vitamin E TPGS as a molecular biomaterial for drug delivery Biomaterials. 2012;33(19):4889-4906. doi: 10.1016/j.biomaterials.2012.03.046

18. Matthew J Webber, John B Matson Vibha K. Tamboli, Samuel I Stupp. Controlled release of dexamethasone from peptide nanofiber gels to modulate inflammatory response Biomaterials. 2012;33(28):6823-6832. doi: 10.1016/j.biomaterials.2012.06.003

19. Yuwei Wang, Kenny K Tran, Hong Shen, David W Grainger. Selective local delivery of rank RNA to bone phagocytes using bone augmentation. Biomaterials. 2012;33(33):8540-8547. doi: 10.1016/j.biomaterials.2012.07.039

20. IJ Macha, S Cazalbou, R Shimmon, B Ben-Nissan, B Milthorpe. Hydroxyapatite/PLA biocomposite thin films for slow drug delivery of antibiotics for the treatment of bone and implant-related infections. Journal of tissue engineering and regenerative medicine. 2015;696:271-276.

21. Fuyin Zheng, Shige Wang, Shihui Wen, Mingwu Shen, Meifang Zhu, Xiangyang Shi. Characterization and antibacterial activity of amoxicillin-loaded electrospun nanohydroxyapatite /poly(lactic-co-glycolic acid) composite nanofibers. Biomaterials. 2013;34(4):1402-1412. doi: 10.1016/j.biomaterials.2012.10.071 